\title{
\begin{tabular}{c} 
Case \\
Report \\
\hline
\end{tabular}
}

\section{Successful Management of Refractory Lethal Coronary Spasm after Off-Pump Coronary Bypass Grafting}

\author{
Soh Hosoba, MD, Tomoaki Suzuki, MD, PhD, Noriyuki Takashima, MD, \\ Takeshi Kinoshita, MD, Satoshi Kuryanagi, MD, Hiromitsu Nota, MD, and \\ Tohru Asai, MD, PhD
}

\begin{abstract}
Coronary artery spasm after coronary artery bypass grafting is a rare cause of acute myocardial infarction. A 68-year-old man who successfully tolerated off-pump coronary artery bypass grafting had a life-threatening spasm at 16 hours postoperatively. Emergent coronary angiography was performed and demonstrated whole vessel spasm of the bilateral coronary arteries and completely patent grafts. Several transcatheter intracoronary injections of vasodilators failed to relieve the spasm completely. After observation in ICU for 4 days with intraaortic balloon pumping and a high dose of catecholamine, cardiac function was re-established and the patient recovered.
\end{abstract}

Keywords: coronary artery spasm, coronary artery bypass grafting complication, off-pump coronary artery bypasses grafting

\section{Introduction}

Coronary artery spasm is an uncommon but known complication of coronary artery bypass grafting (CABG). Off-pump coronary bypass grafting (OPCAB) is considered to be a technique of low invasiveness, ${ }^{1)}$ but is nevertheless associated with the serious complication of perioperative coronary spasm. Because the factors in coronary spasm are not fully understood and the diagnosis is made exclusively by angiography, the true incidence rate remains unclear.

We report a successfully managed rare case presenting with refractory life-threatening coronary spasm after OPCAB.

$\overline{\text { Division of Cardiovascular Surgery, Shiga University of Medical }}$ Science, Otsu, Shiga, Japan

Received: July 25, 2011; Accepted: October 12, 2011

Corresponding author: Soh Hosoba, MD. Division of Cardiovascular Surgery, Shiga University of Medical Science, Seta

Tsukinowacho, Ohtsu, Shiga 520-2192, Japan

Email: sohosoba@belle.shiga-med.ac.jp

(C)2011 The Editorial Committee of Annals of Thoracic and

Cardiovascular Surgery. All rights reserved.

\section{Case Report}

A 68-year-old Asian man with a 9-month history of typical effort angina who developed retrosternal oppression underwent percutaneous coronary intervention (PCI) at the ostium of the left anterior descending artery (LAD) and the first diagonal branch (Dx) and had a stent inserted in the LAD. The patient had been well until two months earlier, when he began to have episodes of anterior and posterior chest pain and dyspnea on light exertion, which was relieved by nitroglycerin. Echocardiography showed almost normal wall motion without valvar malfunction apart from mild aortic insufficiency. His ejection fraction was $60 \%$. Cardiac enzymes were not elevated. A coronary angiography (CAG) was performed. The ostium of the LAD and first Dx was severely restricted, with approximately $90 \%$ stenosis (Fig. 1). The patient was referred to our surgical team as a case of unstable angina pectoris after heparinization. The results of routine laboratory tests were normal with the exception of slight elevation of renal function test values (creatinine: 1.47).

He had been taking daily aspirin till on the operation 

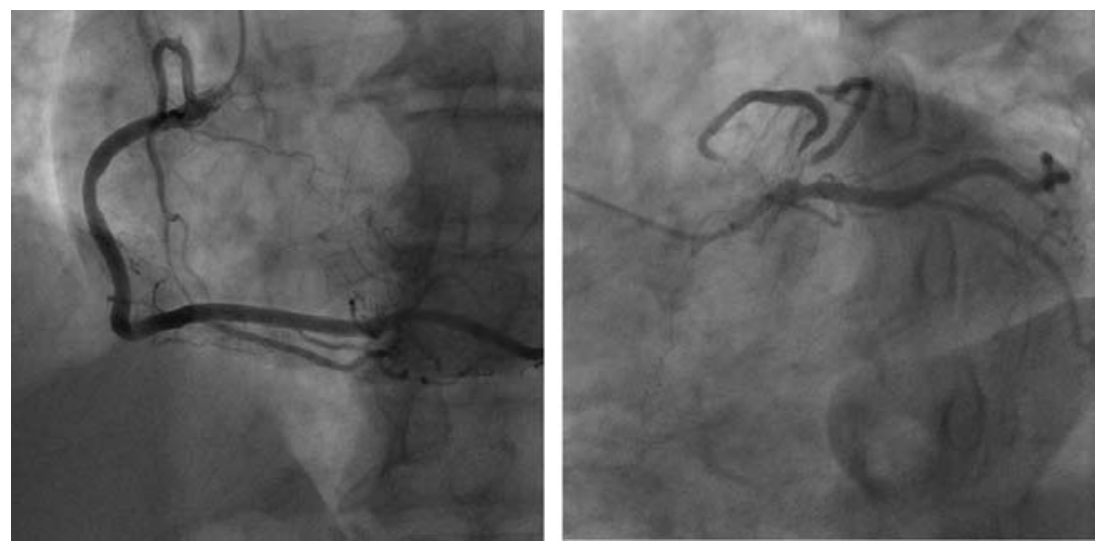

Fig. 1 Coronary angiography showed severe stenosis of the ostium of the left anterior descending artery and the first diagonal branch. The right coronary artery had no stenotic lesion.

day. He did not take any herbal remedies or illicit drugs. The patient underwent an urgent OPCAB. Following medial sternotomy, the bilateral internal mammary arteries (IMA) were harvested in a skeletonized fashion using electrocautery and an ultrasound scalpel. Two deep pericardial stitches were inserted, and gauze pads were placed behind the heart for a clearer view of the LAD. A 2-mm intracoronary shunt (Anastaflow; Intravascular Shunt, Research Medical, Midvale, USA) and a mechanical stabilizer (Octopus stabilizer; Medtronic, Minneapolis, MN, USA) were used. The coronary artery bypasses were made by joining the right IMA to the LAD and the left IMA to the Dx with 7-0 prolene running suture. The graft flows determined by transit time flow measurement were $55 \mathrm{~mL} / \mathrm{min}$ in the right IMA and $38 \mathrm{~mL} / \mathrm{min}$ in the left IMA. Overall operation time was 153 minutes. The operation was completed quickly and uneventfully, and the patient had an uneventful recovery in the ICU. His blood pressure was adequately controlled within 100 to $130 \mathrm{mmHg}$ systolic. He was extubated 9 hours and returned to the general ward 15 hours postoperatively with a 5 milligram of nitroglycerin patch. At this point, the patient complained of back pain and experienced a sudden drop in blood pressure and heart rate. EKG showed STsegment elevation in leads II and III and augmented voltage foot (aVF). The patient was intubated and a catecholamine drip introduced after insertion of a central venous line. Echocardiography showed diffuse and severe hypokinesis globally resembling Takotsubo cardiomyopathy.

The patient was taken to the catheter laboratory after injection of bolus heparin. Emergent CAG showed severe vasospasm of the whole native bilateral coronary arteries (Fig. 2A) and completely patent IMAs (Fig. 2B). After insertion of intra-aortic balloon pumping (IABP), transcatheter administration of vasodilators including nitroglycerin (1.0 mg in total), and a continuous drip of calcium-channel blockers (diltiazem $1 \mathrm{mg}$ ) was applied but produced little improvement. These agents were continued as drip infusion (nitroglycerin $3 \mathrm{mg}$ per hour and diltiazem $2 \mathrm{mg}$ per hour). The electrocardiographic ST segment elevation persisted. The patient was supported with IABP and a high dose of catecholamine (7 gamma of dopamine). The systolic blood pressure of the patient was around 80 to $90 \mathrm{mmHg}$, and pulmonary arterial pressure was 30 to $40 \mathrm{mmHg}$. The heart rate was around 90 . The data of arterial blood gas demonstrated enough oxygenation. We decided to wait and see till the heart muscle recovered from the stun. The patient was placed under deep sedation with simultaneous drip infusion of diltiazem and nitroglycerin. The maximal value of creatinine phosphokinase was $5112 \mathrm{IU} / \mathrm{L}$, that of MB isozyme $818 \mathrm{IU} / \mathrm{L}$ and troponin $394 \mathrm{IU} / \mathrm{L}$. After 3 days of observation in the ICU, cardiac function gradually recovered. On the fourth postoperative day, IABP was successfully discontinued with 5 gamma of dobutamine and the patient extubated. Coronary angiography, repeated on the 13th postoperative day, demonstrated non-spastic native coronary arteries (Fig. 3A) and patent grafts (Fig. 3B). Echocardiography on the 8 th postoperative day demonstrated ejection fraction of $50 \%$. The patient was discharged in good clinical condition on the 21 st postoperative day and remains symptom-free at 3 months postoperatively. 

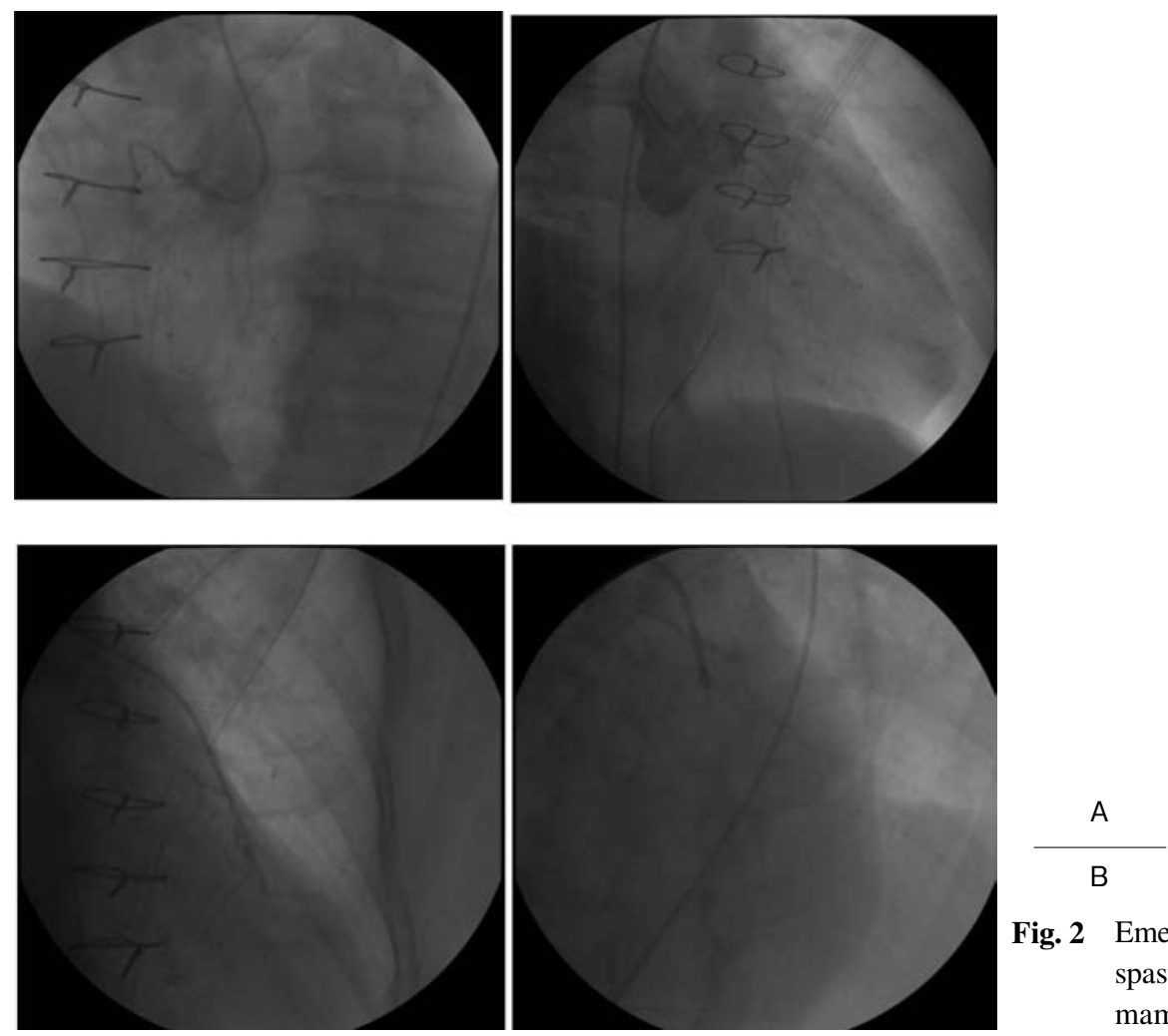

Fig. 2 Emergent coronary angiography showed severely spastic native coronary arteries (A). The internal mammary arteries were all patent $(\mathbf{B})$.
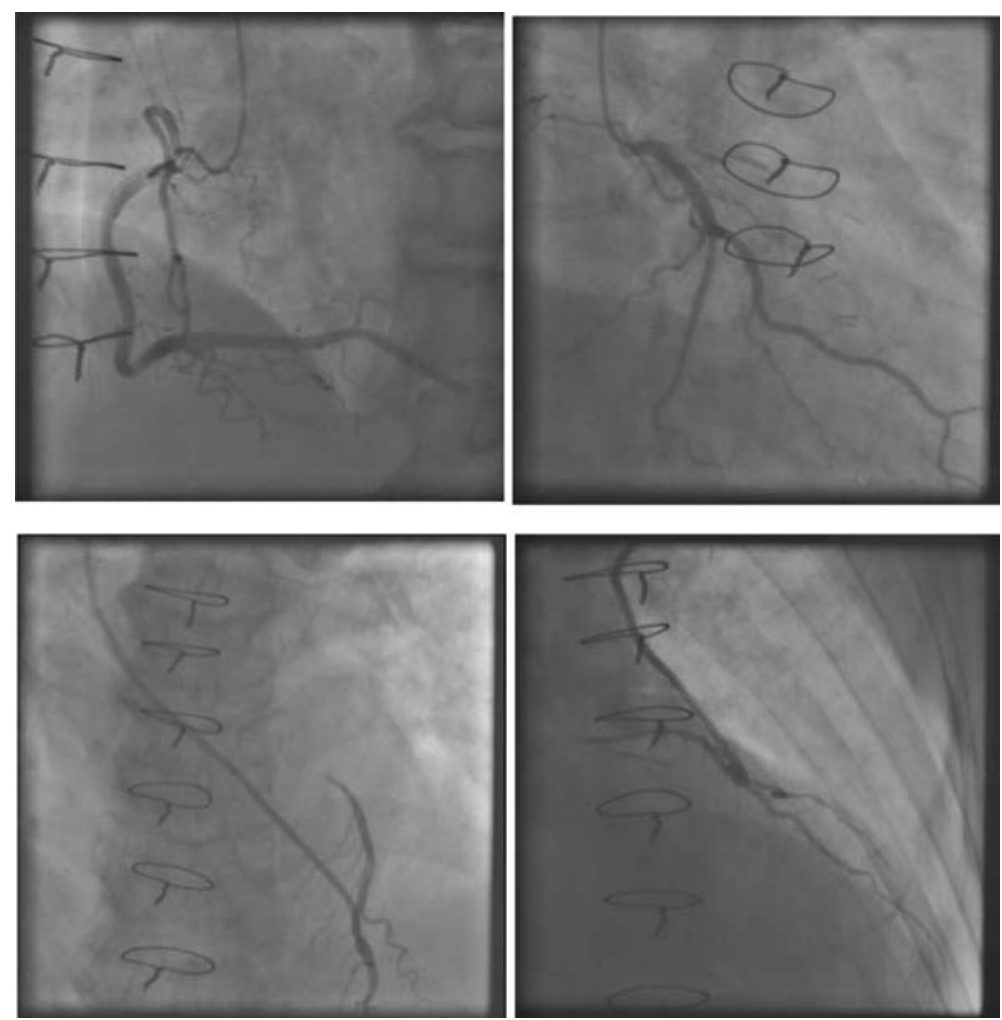

A

B

Fig. 3 Postoperative coronary angiography showed non spastic coronary arteries (A) and patient internal mammary arteries (IMA) (B). The left descending artery was totally supplied with right IMA. The first diagonal branch was supplied with left IMA. 


\section{Discussion and Conclusion}

Coronary artery spasm after CABG is relatively rare but can be a life-threatening complication. It may occur during surgery ${ }^{2)}$ or in the immediate postoperative period of CABG. ${ }^{3,4)}$ It may occur on a manipulated or non-manipulated vessel.

The possible causes of coronary spasm are still undetermined. Many factors have been proposed to account for this pathology, including manipulation during operation, smoking, high endogenous or exogenous catecholamine levels, hypothermia, hypocapnia, and hypomagnesemia. ${ }^{3,5)}$ The patient was a non smoker, had a magnesium injection soon after the operation, had the vasospasm on a non manipulated site, and had a quick operation with no catecholamine infusion.

The gold standard for revealing coronary spasm is CAG. Also deserving of attention during the postoperative phase is EKG change, Takotsubo-like movement in echocardiography, and patient complaint. Urgent CAG and prompt treatment with intraluminal injection of vasodilator are consideredbeing essential. ${ }^{7)}$

In some settings, coronary spasm is refractory to vasodilating agents and induces malignant arrhythmia and circulatory collapse. To treat this condition, many medications such as magnesium Rho-kinase inhibitors ${ }^{8)}$ or nifedipine ${ }^{9)}$ have been proposed. Stent implantation is also considered being an option. ${ }^{10)}$ In this case, however, the whole coronary arteries were spastic and there was no indication of stent implantation. We believe that vasospasm is reversible and that, if the circulation status has not collapsed, a-wait-and-see policy with supportive agents can be safe and effective. In the present case, the myocardial injury was massive and greater than in previous reports, ${ }^{11)}$ with a creatinine phosphokinase value of 5112 , but the patient survived.

In conclusion, we report a case that highlights the importance of evaluating the risk of postoperative vasospasm. Lethal coronary artery spasm refractory to intracoronary injection can occur after uneventful CABG.

\section{Disclosure Statement}

We have no financial or other interest.

\section{References}

1) Puskas JD, Williams WH, Duke PG, et al. Off-pump coronary artery bypass grafting provides complete revascularization with reduced myocardial injury, transfusion requirements, and length of stay: a prospective randomized comparison of two hundred unselected patients undergoing off-pump versus conventional coronary artery bypass grafting. J Thorac Cardiovasc Surg 2003; 125: 797-808.

2) Bossert T, Bittner HB, Gummert JF, et al. Coronary artery spasm of the native right coronary artery during off-pump coronary surgery of the left coronary artery system. Clin Res Cardiol 2006; 95: 115-8. Epub 2006 Jan 16.

3) Minato N, Katayama Y, Sakaguchi M, et al. Perioperative coronary artery spasm in off-pump coronary artery bypass grafting and its possible relation with perioperative hypomagnesemia. Ann Thorac Cardiovasc Surg 2006; 12: 32-6.

4) Lin CY, Weng ZC, Loh SH, et al. Coronary artery spasm after off-pump coronary artery bypass grafting. ANZ J Surg 2007; 77: 126-9.

5) Buxton AE, Goldberg S, Harken A, et al. Coronary-artery spasm immediately after myocardial revascularization: recognition and management. $\mathrm{N}$ Engl J Med 1981; 304: 1249-53.

6) Sugiishi M, Takatsu F. Cigarette smoking is a major risk factor for coronary spasm. Circulation 1993; 87: 76-9.

7) He GW, Fan KY, Chiu SW, et al. Injection of vasodilators into arterial grafts through cardiac catheter to relieve spasm. Ann Thorac Surg 2000; 69: 625-8.

8) Inokuchi K, Ito A, Fukumoto Y, et al. Usefulness of fasudil, a Rho-kinase inhibitor, to treat intractable severe coronary spasm after coronary artery bypass surgery. J Cardiovasc Pharmacol 2004; 44: 275-7.

9) Prida XE, Gelman JS, Feldman RL, et al. Comparison of diltiazem and nifedipine alone and in combination in patients with coronary artery spasm. J Am Coll Cardiol 1987; 9: 412-9.

10) Kaku B, Ikeda M, Kato H, et al. Coronary artery multistenting in the treatment of life-threatening refractory coronary spasm after coronary artery bypass grafting. Int Heart J 2007; 48: 379-85.

11) Ueno $T$, Ikeda $K$, Nakashima A. Vasodilator resistant lethal spasm after uncomplicated off-pump coronary surgery. Asian Cardiovasc Thorac Ann 2006; 14: 15860. 